\title{
The Population of Debris Discs Orbiting Subgiants
}

\author{
Amy Bonsor ${ }^{1}$, Grant M. Kennedy ${ }^{2}$, Justin R. Crepp ${ }^{3}$, \\ John A. Johnson ${ }^{4}$, Mark C. Wyatt ${ }^{2}$, Bruce Sibthorpe ${ }^{5}$ and \\ Kate Y. L. $\mathrm{Su}^{6}$ \\ ${ }^{1}$ UJF-Grenoble 1 / CNRS-INSU, Institut de Plantologie et d'Astrophysique de Grenoble \\ (IPAG) UMR 5274, Grenoble, F-38041, France \\ ${ }^{2}$ Institute of Astronomy, University of Cambridge, Madingley Road, Cambridge CB3 OHA, UK \\ ${ }^{3}$ Department of Physics, University of Notre Dame, 225 Nieuwland Science Hall, Notre Dame, \\ IN 46556, USA \\ ${ }^{4}$ Department of Astronomy, California Institute of Technology, 1200 E. California Blvd., \\ Pasadena, CA 91125, USA \\ ${ }^{5}$ SRON Netherlands Institute for Space Research, Zernike Building, P.O. Box 800, 9700 AV \\ Groningen, The Netherlands \\ ${ }^{6}$ Steward Observatory, University of Arizona, 933 N Cherry Ave., Tucson, AZ 85721 \\ email: amy.bonsor@gmail.com
}

\begin{abstract}
Whilst debris discs orbiting main-sequence stars are well studied, very little is known regarding their fate when the star evolves onto the giant branch. For intermediate mass (A-type) stars, giants provide a unique opportunity to detect planets using the radial velocity technique, otherwise prohibited by high jitter levels and rotationally broadened lines in main-sequence intermediate mass (A-type) stars. Such stars can provide key insights into the structure of planetary systems around intermediate mass stars. In our Herschel OT1 program (PI Bonsor) we searched for the presence of debris discs orbiting a sample of 36 subgiants, half of which have $\mathrm{RV}$ detected companions. Our best detection is the resolved debris disc orbiting $\kappa \mathrm{CrB}$.
\end{abstract}

Keywords. (stars:) planetary systems,(stars:) circumstellar matter

\section{Kappa Cr B (Bonsor et al., 2013)}

Our best detection, Kappa Cr B, provides the first resolved images of a debris disc orbiting an evolved star. Kappa Cr B is also orbited by two companions. The structure of this planetary system is constrained from the Herschel images (Fig. 1, alongside detection limits on the orbital parameters of these companions, obtained using further RV monitoring (Keck I HIRES) and AO imaging (Keck II NIRC2)). Kappa Cr B is an intriguing example of a planetary system where both planets and dusty debris have been detected and in which interactions between them may be critically important. See Bonsor et al., 2013 for more details.

\section{The Herschel Survey (Bonsor et al., in prep)}

Thirty eight subgiants were searched for excess emission with Herschel PACs at 100 $\mu \mathrm{m}$ and $160 \mu \mathrm{m}, 20$ planet-hosts and 16 control stars. The aim was to search for a correlation between planet-hosts and non-planet host stars. Excess emission, consistent with a debris disc, was found for $4 / 36(2 / 36)$ of the subgiants in our sample at $100 \mu \mathrm{m}$ $(160 \mu \mathrm{m})$. Three of the detections are planet-hosting stars. See Bonsor et al. (in prep) for more details. 

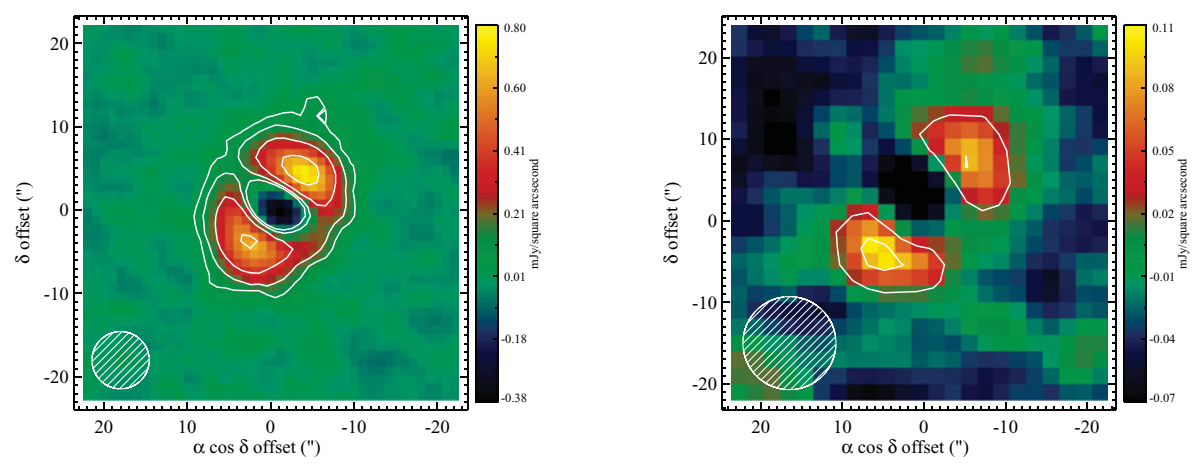

Figure 1. The Herschel images of the $\kappa \mathrm{CrB}$ debris disc (stellar-subtracted) at $100 \mu \mathrm{m}$ (left) and $160 \mu \mathrm{m}$ (right) (Bonsor et al., 2013)
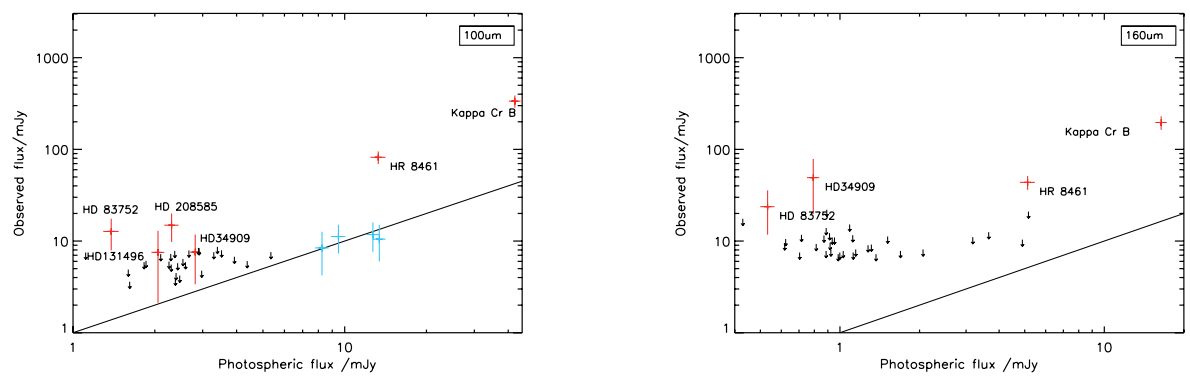

Figure 2. The detections in our Herschel survey. The photospheric flux, predicted using stellar models compared to the observed flux, at $100 \mu \mathrm{m}$ (left) and $160 \mu \mathrm{m}$ (right). The red (blue) points show $3-\sigma$ detections of a disc (the star), with $3-\sigma$ error bars. The stellar photosphere is too faint at $160 \mu \mathrm{m}$ to have been detected by any of our observations.

\section{Reference}

Bonsor, A.; Kennedy, G. M., Crepp, J. R., Johnson, J. A., Wyatt, M. C., Sibthorpe, B., \& Su, K. Y. L. 2013, MNRAS, 431, 3025-3035 